\title{
Basic Residue Clusters in Intrinsically Disordered Regions of Peripheral Membrane Proteins: Modulating 2D Diffusion on Cell Membranes
}

\author{
Miquel Pons (D)
}

Citation: Pons, M. Basic Residue Clusters in Intrinsically Disordered Regions of Peripheral Membrane Proteins: Modulating 2D Diffusion on Cell Membranes. Physchem 2021, 1, 152-162. https://doi.org/10.3390/ physchem 1020010

Academic Editors: Jacinto Sá and Sergei Manzhos

Received: 26 May 2021

Accepted: 1 July 2021

Published: 19 July 2021

Publisher's Note: MDPI stays neutral with regard to jurisdictional claims in published maps and institutional affiliations.

Copyright: (C) 2021 by the author. Licensee MDPI, Basel, Switzerland. This article is an open access article distributed under the terms and conditions of the Creative Commons Attribution (CC BY) license (https:/ / creativecommons.org/licenses/by/ $4.0 /)$.
Biomolecular NMR Laboratory, Department of Inorganic and Organic Chemistry, Universitat de Barcelona, Baldiri Reixac, 08028 Barcelona, Spain; mpons@ub.edu

\begin{abstract}
A large number of peripheral membrane proteins transiently interact with lipids through a combination of weak interactions. Among them, electrostatic interactions of clusters of positively charged amino acid residues with negatively charged lipids play an important role. Clusters of charged residues are often found in intrinsically disordered protein regions, which are highly abundant in the vicinity of the membrane forming what has been called the disordered boundary of the cell. Beyond contributing to the stability of the lipid-bound state, the pattern of charged residues may encode specific interactions or properties that form the basis of cell signaling. The element of this code may include, among others, the recognition, clustering, and selective release of phosphatidyl inositides, lipid-mediated protein-protein interactions changing the residence time of the peripheral membrane proteins or driving their approximation to integral membrane proteins. Boundary effects include reduction of dimensionality, protein reorientation, biassing of the conformational ensemble of disordered regions or enhanced 2D diffusion in the peri-membrane region enabled by the fuzzy character of the electrostatic interactions with an extended lipid membrane.
\end{abstract}

Keywords: intrinsically disordered proteins; peripheral membrane proteins; charge clusters; lipidmediated protein interactions; reduction of dimensionality; enhanced diffusion; crowding

\section{Introduction}

The functional integration of the cell with its environment starts in the highly dynamic cell boundary where the interaction of lipids and membrane proteins ensure signal transduction, cell-cell communication, extracellular adhesion, and a myriad of physiological responses of the whole cell initiated at the cell surface.

The sharp academic division between soluble and (integral) membrane proteins has obscured the importance of peripheral membrane proteins (PMP). While integral membrane proteins are permanently associated to membranes, PMPs interact reversibly with a single membrane leaflet or with the cytoplasmic domains of integral membrane proteins. These transient interactions enable the distribution of PMPs among alternative subcellular compartments, or membrane subdomains, facilitate fast lateral diffusion on the membrane surface, and play a key role in cell signalling.

Multiple weak interactions mediate the dynamic interactions between PMPs and the other membrane component (lipids, integral membrane proteins) and the underlying cytoskeleton. Dynamics often include liquid-like exchange of the interacting lipids as well as conformational freedom on the proteins. Lipids play an active role in signaling, supported by the presence of at least 800 distinct species in a typical mammalian cell membrane, responding to culture conditions and cell [1]. The abundance of intrinsically disordered regions permanently or transiently bound to the membrane is one characteristic of the membrane periphery, described as the "disordered boundary of the cell" [2]. In fact, intrinsically disordered regions (IDR) longer than 30 residues are more abundant in the subset of intrinsic membrane proteins $(57 \%)$ than in the complete human proteome $(34 \%)$, 
suggesting that around $45 \%$ of IDRs belong to proteins with transmembrane regions. The combined abundance of myristoylated IDPs such as MARCKS (myristoylated alanine rich $C$ kinase substrate), the alpha subunits of $G$ proteins, and Src family kinases represents $15 \%$ of that of actin, the major component of the cytoskeleton [3]. The importance of intrinsic disorder in membrane cell signaling has been recently reviewed [4].

This perspective paper is focused on PMPs and is inspired in recent findings on clustering and reversible versus irreversible membrane anchoring by the non-receptor tyrosine kinase Src and the role played by a cluster of basic residues. Clusters of basic residues are also common in the intrinsically disordered juxtamembrane region of integral membrane proteins and adjacent to lipid modification sites of other PMP, such as members of the RAS family of GTPases.

\section{Peripheral Membrane Proteins}

PMP interacts reversibly with the surface of membranes and, therefore, may be found in soluble form in the cytosol. The term 'amphitropic' is sometimes used to reflect their multiple localization [5].

A dataset of experimentally annotated human peripheral membrane proteins in Uniprot-Swiss-Prot containing 277 plasma membrane proteins has been used to define the human peripherome, including protein-protein interaction clusters associated with the membrane surface [6]. The Orientation of Proteins in Membranes (OPM) database contains 1566 entries classified as monotopic/peripheral protein [7]. Only a small fraction of these bind irreversibly [8]. PMPs represents close to one-third of the proteins in the OPM database.

The actual number of peripheral membrane proteins is larger since peripheral membrane proteins anchored to membranes through intrinsically disordered regions that lack a well-defined structure, such as c-Src, and the other Src Family Kinase members are not included in the OPM database.

Binding of PMP results from the combination of non-specific hydrophobic interactions, specific interactions with lipids, and electrostatic contributions with charged lipid headgroups. Some of the PMP are fatty acylated or prenylated, interacting directly with the lipid bilayer by a combination of lipid insertion and electrostatic interactions [9].

Electrostatic interactions can be non-specific, i.e., independent of the nature of the charged groups in the protein and lipids, or specific, involving a direct interaction between the side chains of basic residues and lipid phosphate groups. Arginine containing peptides bind 10-fold stronger than their lysine analogues to negatively charged membranes, in spite of having the same number of charges [10].

The special relevance of electrostatic interactions in PMP ultimately derives from the asymmetric distribution of charged lipids in membranes (Figure 1) [11]. 


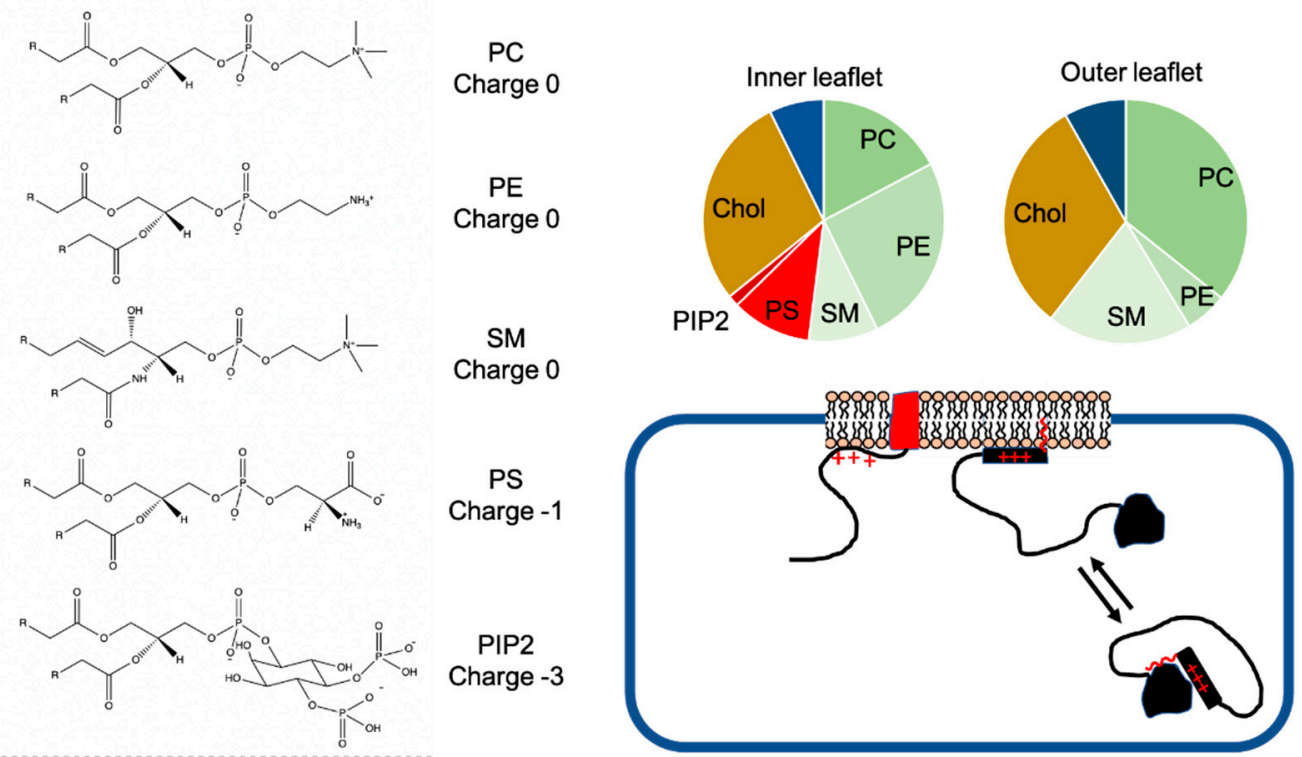

Figure 1. Headgroup structure and relative abundance of lipids in eukaryotic cell membranes. PC: phosphatidylcholine; PE: phosphatidylethanolamine; SM: sphingomyelin; PS: phosphatidylserine; PIP2: phosphatidylinositol-4.5-bisphosphate. The cartoon represents two types of positively charged disordered regions: those forming the cytoplasmatic end of an integral membranme proteins (such as receptor tyrosine kinases (RTK)) and a peripheral membrane protein with a myristoylated and positively charged membrane anchoring domain (such as c-Src) that can dissociate from the membrane, possibly by alternative burying of the acyl chain in a pocket in a globular domain.

\section{Asymmetric Distribution of Charged Lipids in Membranes}

Negatively charged phospholipids (phosphatidylserine and phosphatidylinositol) are concentrated in the cytoplasmic leaflet of the lipid bilayer in eukaryotic cells. This asymmetric lipid distribution creates a potential within the bilayer and a difference in surface potentials, including the contributions from counterions that are attracted, and coions that are repelled at both sides. The resting potential at the surface of a typical differentiated eukaryotic membrane is between $-40 \mathrm{mV}$ and $-80 \mathrm{mV}$, but it can be much lower in undifferentiated cells or in human erythrocytes [12]. The ionic atmosphere screens the electric potential with an exponentially decaying law defining the Debye length, which is around $1 \mathrm{~nm}$ for a $100 \mathrm{mM}$ solution of a monovalent salt. The surface potential is well described by the Gouy-Chapman theory that assumes a uniform charge density on the membrane charge surrounded by point charges. Ions with multiple charges will be concentrated close to the membrane surface. A substrate with six positive charges would be concentrated by three orders of magnitude near the membrane interface, potentially affecting reactivity of enzyme substrates or self-association processes.

Interestingly, lipid asymmetry, even involving only zwitterionic lipids, can also generate an intramembrane potential in the absence of salt, as shown by molecular dynamic calculations [13], highlighting the effect of dipole derived potentials.

The simplifying assumptions of the Gouy-Chapman theory are reasonable assumptions for small ions, but additional contributions apply for large molecules, such as proteins with clusters of charged residues. While only charges within the Debye length from the membrane surface will experience a significant potential, globular proteins can become oriented in the vicinity of membranes. The remarkable symmetry of charged residues of kaliotoxin around the principal axis of its inertia tensor probably facilitates the orientation of the electrostatically driven approach of the toxin towards the mouth of the potassium channel [14].

The strong electrostatic field in the vicinity of the membrane $\left(10^{5} \mathrm{Vcm}^{-1}\right)$ [11] can substantially change the conformational landscape, triggering additional interactions, especially in intrinsically disordered proteins with multiple charged residues [2]. 
Interestingly, various toxins interacting with the outer leaflet, enriched in phosphatidylcholine headgroups, have tyrosine residues participating in cation-p interactions with the positively charged choline headgroup [15,16]. These weak, opportunistic, interactions contrast with the specific interactions found in choline [17] or three-methyl lysine receptors [18].

There is an obvious parallelism between the weak, rapidly exchanging interactions of PMP across a large number of lipid sites, and the rapidly exchanging interactions binding together the disordered components in fuzzy complexes [19]. Interestingly, the membrane component represents a near-infinite plane and exchange between lipid sites, as well as long range electrostatic interactions, enable fast lateral diffusion constrained to the immediate neighborhood of the lipid boundary.

\section{Positively Charged Disordered Regions in the Vicinity of Lipid Anchors Can Arise from Basic Residue Clusters or Depletion of Acidic Residues}

Myristoylation or attachment of a farnesyl group are common post-translational modification associated to membrane anchoring. However, insertion of the 14-carbon fatty acid in a lipid bilayer accounts for barely $8 \mathrm{kcal} \mathrm{mol}^{-1}$ binding energy [20], corresponding to an affinity constant $\mathrm{K}_{\mathrm{a}} \cong 10^{4} \mathrm{M}^{-1}$; thus, it is not enough to ensure stable membrane anchoring and a second membrane anchoring element is needed, either by a cysteine attached palmitoyl group or a polybasic peptide segment. The electrostatic interaction of basic peptides with acidic lipids is estimated to be around $1.4 \mathrm{kcal} \mathrm{mol}^{-1}$ per positive charge [21]

The electrostatic contribution has been extensively demonstrated by the work of McLaughlin and coworkers, including its modulation by post-translational phosphorylation in the vicinity of basic residue clusters. [11,20,22] The polybasic element may be located near the myristoylation site, as in the case of c-Src, or more distant, such as in MARCKS, and HIV-1 Nef and Gag proteins.

The abundance of basic residues contributing to the stability of membrane binding by peripheral membrane proteins is often assumed to be a general motif but statistical surveys in a number of databases suggests that this general assumption needs to be qualified.

A statistical analysis of the predicted membrane orientations of peripheral/monotopic proteins in the OPM database [16], which is based on structural data on globular domains, shows that basic residues are not overrepresented in these proteins. There is a slightly higher density of basic residues in the region of the lipid phosphate headgroup, but it is not uncommon that proteins in the OMP have three or less basic residues within $10 \AA$ from the end of the hydrocarbon region.

A database of lipid-binding intrinsically disordered proteins (LBID) [23] contains entries in the DisPro database [24] with keywords associated to lipid binding. It contains 119 entries, which represent $15 \%$ of DisProt. The abundance of basic residues in the disordered regions of the LBID proteins is $11.9 \%$, very similar to that of the entire DisProt database $(12.7 \pm 0.8 \%)$. The 277 proteins in the human peripherome database have a similar abundance of basic residues $(12.3 \%)$.

Focusing the analysis on the ensemble of disordered regions adjacent to myristoylation sites, we analyzed the sequences of the initial 16-peptides of 66 myristoylated proteins published by Resh [25] (Figure 2) and a recent survey by Castrec et al. [26] collected the initial 8-peptides of 364 human myristoylated proteins. The abundance of basic residues in the two surveys are $13.3 \%$ and $12.0 \%$, again not very different from the expected values for a disordered region. Interestingly, a significant depletion in acid residues is observed close to the myristoylation site: Aspartic or glutamic acid represent only $9.4 \%$ of the residues in the 16-peptides and 3.7\% in the 8-peptides, in contrast to the $13.0 \pm 0.7 \%$ abundance of these residues in the DisProt database. 
<smiles>CC(=O)C(N)CCCNC(=N)N</smiles><smiles>CC(=O)C(N)CCCC[NH3+]</smiles><smiles>CC(=O)C(N)CCC(=O)O</smiles>

Glutamic acid (E)<smiles>CC(=O)C(N)CC(=O)O</smiles>

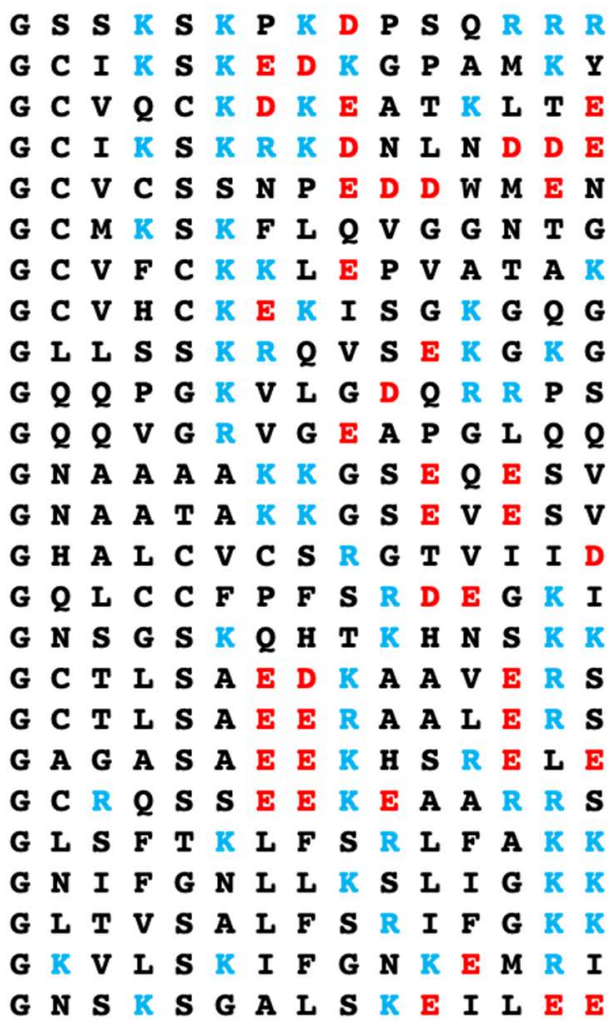

Figure 2. Left. Structure of the charged amino acid residues. Right. Representative sequences of the initial 15 residues (after removal of the initial methionine) of myristoylated proteins listed by Resh et al. [24] with positively and negatively charged residues marked in blue and red, respectively.

Prenylation close to the $\mathrm{C}$-terminus is an alternative membrane anchoring mechanism that, like myristoylation, is often associated with an enrichment in basic residues. The analysis of the 35-residue C-terminal sequences containing an experimentally identified prenylation site obtained in a large-scale profiling of prenylated proteins in the mouse macrophage line RAW264.7 [27] shows an enrichment in basic residues (16.2\%) and only a small decrease in the abundance of acid residues $(11.7 \%)$ with respect to the abundance of these residues in the DisProt database.

Thus, the overall enrichment in positive charges in the disordered regions located in the vicinity of covalent lipid anchors (N-terminal myristoyl and C-terminal prenyl) mainly originates from a decrease in the number of negative charges close to the $\mathrm{N}$-terminus but from an enrichment in basic residues close to the C-terminus.

In the above statistical analysis, myristoylated or prenylated regions containing additional lipid anchors (e.g., palmitoyl) were not differentiated from those containing a single acylation site, in spite of the fact that thermodynamic considerations on the stability of the membrane-bound forms suggest that the electrostatic contribution may be negligible when two or more hydrophobic groups are inserted in the lipid bilayer. However, while myristoylation is predominantly co-translational, palmitoylation takes place on the membrane surface, thus electrostatic interactions facilitate the initial binding of the single acylated form, even if they are no longer needed to ensure stable membrane binding after a second acyl group is attached.

The increased positive charge density in disordered regions located near the membrane surface may play additional roles beyond the pure electrostatic contribution to the stability of the membrane bound state. At least two mechanisms depending on clusters of basic residues have been described so far: the modulation of the local concentration of acidic 
lipids, specially the highly charged but not highly abundant phosphoinositide phosphates, and the lipid-mediated clustering of peripheral membrane proteins.

\section{Other Roles of Positively Charged Disordered Regions Bound to Membranes: PIP Modulation}

Multiple charged species, lipids, and proteins, can mutually affect their lateral distribution. As discussed by McLaughlin [11], extending the Bjerrum theory of ion association to two dimensions, 3:3 electrolytes are expected to be strongly associated, and 3,4-phosphatidylinositide phosphate, with a net charge of -3 can be sequestered by positively charged MARKS, and released upon phosphorylation, acting as a PIP-modulin [28].

Receptor tyrosine kinases (RTK) contain a ligand binding ectodomain, a single transmembrane helix linked, and an intracellular region starting with an intrinsically disordered region (juxtamembrane) followed by the catalytic domain. The juxtamembrane region is enriched in basic residues. In the ensemble of 20-residue segments following the transmembrane helix of the 58 human RTK, basic residues represent $24.5 \%$ and acidic residues are just $11.0 \%$. The juxtamembrane regions have a regulatory role, mediated by interactions with the catalytic domain in numerous RTKs, receptor dimerization, and interaction with anionic lipids. Epidermal Growth Factor Receptor (EGFR) juxtamembrane peptides were found to bind to PIP2 using surface plasmon resonance [29] and to induce clustering of PIP2 in vesicles [28]. The role of basic residues in the juxtamembrane region was confirmed by charge neutralizing mutations abolishing the interaction. Clustering of phosphatidyl serine and PIP2 was observed in molecular dynamic [30] and multiscale simulations [31] of full length EGFR. Coarse-grained simulations indicate the reorganization of acidic lipids, specially PIP2, in shells around the juxtamembrane region as a result of interactions with clusters of basic residues [32].

\section{Self-Association of Peripheral Membrane Proteins Involving Basic Residue Clusters}

While basic protein regions can sequester and concentrate minor acidic lipids, membrane proteins with basic residue clusters can also be clustered by electrostatic interactions with lipids. Clustering of syntaxin-1A by PIP2 was observed experimentally and reproduced in molecular dynamics simulations that showed PIP2 acting as "charge-bridge" bringing together various syntaxin-1A molecules [33]. PIP2-mediated syntaxin-1A clusters are different from those that are observed in the presence of cholesterol and zwitterionic dioleoyl phosphatidyl choline. In the latter, syntaxin-1A clusters are induced by lipid-phase separation. Syntaxin-1A in PIP2 induced electrostatic clusters remains highly mobile by fluorescence recovery after photobleaching, while the cholesterol induced clusters are essentially immobile.

Electrostatic ordering of extended peptide segments on the surface of lipid membranes can facilitate other protein clustering effects. Hädicke et al. observed the formation of anti-parallel $b$-sheet by model cationic peptides with alternating lysine and non-charged residues [34]. Sinthuvanich et al. engineered a cationic peptide SVS-1 $\left(K_{V K V K V K V}{ }^{\mathrm{D}}{ }^{\mathrm{L}} \mathrm{PTKVKVKVK}-\mathrm{NH} 2\right)$, with anticancer activity, that in solution presents a random coil conformation but folds into a $\beta$-hairpin structure on the negatively charged membrane surface [35]. The outer leaflet of cancer cells, in contrast to healthy cells, tends to be negatively charged. These peptides form random coils in solution but adopt an extended structure because of charged residues in alternating sequence positions being directed towards the charged lipid surface, facilitating the formation of a $\beta$-structure with hydrogen bonds running parallel to the membrane surface. Thus, in contrast to the lipids acting as charge-bridges, direct peptide-peptide interactions occur, in spite of the potential electrostatic repulsion. Presumably, electrostatic repulsion is screened by partial insertion of the charged side chains into the lipid headgroup region.

Alternating basic residue clusters are found in the proximity of myristoylation and prenylation sites of peripheral membrane proteins. Of the 364 proteins in the human mystoylome [26], five proteins contain a cluster formed by three alternating lysines 
$\mathrm{K}-\mathrm{x}-\mathrm{K}-\mathrm{x}-\mathrm{K}$ in the initial eight residues. Two of them are the SFKs Src and Lyn, two are integrated viral endoproteins HERV-K 12q14.1 and 3q12.3, and the fifth is isoform 2 of the spermatogenesis associated protein 33. Similar clusters of alternating basic residues are found in C-terminal farnesylated K-Ras (KHKEK and KSRTR) and protein tyrosine phosphatases PTP4A1 and PRP4A2 (KYRPKMRLRFR/K). The crystal structure of PTP4A1 (PDB 1rxd) [36] show the expected extended structure with positively charged side chains pointing to the same side.

The alternating lysine cluster in the SH4 domain of Src is responsible for the formation of a persistently membrane-bound dimer detected by Surface Plasmon Resonance [37] and Single molecule fluorescence [38]. These dimers represent only a small fraction (less than $10 \%$ ) of membrane-bound Src, but they are not dissociated upon extensive washing, unless a detergent is added. While short myristoylated peptides containing the initial nine residues form oligomers, constructs including the adjacent Unique domain only form dimers, suggesting a physiological role for this process, as it is well known that Src is activated by trans-autophosphorylation $[39,40]$ and membrane induced dimerization would result in a persistently activated signaling species.

In contrast, to the small population of persistently bound dimer, binding and dissociation of monomeric Src is fast. The presence of a myristoyl binding site in the SH3 domain of Src is an additional element facilitating membrane detachment and dissociation [41].

Relocalization between membrane compartments or between membranes and the cytosol is a defining characteristic of peripheral membrane proteins. However, the presence of alternative membrane-bound forms of the same protein, with dramatically distinct dissociation rates suggest that modulation of lateral mobility, and not simply the relative populations of the free and bound forms may be an important, although not always well appreciate characteristic of PMP.

\section{3D versus $2 \mathrm{D}$}

The obvious singularity of processes taking place in, on or around the membrane, as compared with interactions occurring in the bulk of the cytoplasm, is the reduction of dimensionality from 3D to 2D. This subject has been discussed extensively and involves three, more or less independent aspects:

(i) Local concentration: the same number of proteins distributed uniformly in the interior of the cell or restricted to the narrow shell around the membrane would result in a much larger concentration in the vicinity of the membrane. If we assume a cell with a radius of $10 \mathrm{~mm}$ and a submembrane layer of around $3 \mathrm{~nm}$, the increase in concentration is a factor of 1000 . The increased concentrations affect the associationdissociation equilibrium with other partners or that of self-association and also affects the diffusion-driven mechanism of such encounters.

(ii) As first suggested by Adam and Delbrück [42], reduction of dimensionality is expected to reduce the time between random encounters between two species diffusing in 2D. A similar argument has been used to explain how the high efficiency transcription factors find their target sequences in DNA using 1D searches [43].

(iii) Anisotropic interactions: a non-spherical intracellular domain physically tethered to the membrane surface or experiencing strong anisotropic interactions (e.g., electrostatic) may become optimally oriented to present complementary contact points to other membrane anchored components. An example is provided by the formation of antiparallel b-sheet by peptides with alternating basic and neutral residues interacting electrostatically with anionic lipids [34].

\section{Crowding and Restricted 2D Diffusion. Can Be Faster?}

The effect of the reduction of dimensionality on diffusion-controlled reaction rates in membranes has been questioned by pointing out that the lateral diffusion of typical integral membrane proteins $\left(10^{-9}-10^{-10} \mathrm{~cm}^{2} / \mathrm{s}\right)$ [44] is much slower than the typical 3D 
diffusion in the cytoplasm $\left(10^{-8} \mathrm{~cm}^{2} / \mathrm{s}\right)$ [45] and may offset the shorter average distances between objects diffusing in 2D than in 3D [46].

The diffusion coefficient $(\mathrm{D})$ in two-dimensions of integral membrane proteins scales with the protein radius $(R)$ as $\ln (1 / R)$, as predicted by the classical hydrodynamic SaffmanDelbrück model [47] when $R$ is small and the protein is diluted. For larger membrane inserted objects, the dependency approaches a Stokes-like form in which the diffusion coefficient is proportional to $(1 / \mathrm{R})[48,49]$. Recently, an analytical function using a pointlike form was found to correctly predict the diffusion of proteins interacting with only one or both lípid leaflets, including the possible deformation from planarity of the lípid bilayer [50].

Crowding effects are predicted to strongly affect diffusion of proteins in and in the vicinity of membranes. Surprisingly, molecular dynamics simulations predict crowding effects of opposite signs for integral and peripheral membrane proteins.

High densities of integral membrane proteins are very common in biological cells, with average in-plane distances between proteins of only a few nanometers. Coarse-grained molecular dynamics simulations under membrane crowding conditions reflecting frequent protein densities covering around $20 \%$ of the membrane surface, equivalent to ratios of less than 100 lipids per protein, show a strong decrease in lateral diffusion and a similar transition from a Saffman-Delbrück to a Stokes-like scaling of the diffusion coefficients with the particle radius, apparently arising from geometric arguments [51].

PMP can diffuse in 2D much faster than integral membrane proteins [52]. Ziemba and Falke showed that lateral diffusion of PMP on supported lipid bilayers was dominated by the frictional drag of lipids to which the PMP was bound to, in a protein-free aqueous medium.

Crowding on the cytoplasm, however, could strongly restrict the diffusion of the cytoplasmic region. However, based on molecular dynamic calculations, it has been recently suggested that proteins not specifically interacting with lipids are preferentially excluded from the membrane surface, increasing the effective diffusion of proteins located next to the lipid surface, with respect to larger distances, representative of the bulk cytoplasm [53].

Fast 2D diffusion has also been observed next to surfaces covered by intrinsically disordered phenylalanine-glycine nucleoporins [54], and explained by the capacity of intrinsically disordered proteins to undergo fuzzy interactions involving transient exchange between multiple weak interactions. Thus, the cytoplasm layer close to the membrane surface may represent a "fast diffusion" region.

Biological membranes are not homogeneous and are effectively partitioned into compartments, of a few hundred nanometers of diameter, delimited by immobilized membrane proteins contacting the underlying cytoskeleton [55] or by lipid domain boundaries, formed by lipid phase separation or nucleated by protein clusters. Apparent diffusion coefficients effectively depend on the time and length scale observed.

However, since compartments are mostly restricted to the protein-rich lipid membrane surrounded by a relatively obstacle free cytoplasm layer, a combination of fast and slow diffusion can be achieved by some PMPs by fast dissociation-reassociation from the membrane, providing an enhanced exploration of the membrane surface.

Although 2D diffusion can be very efficient to recurrently explore a small area of the membrane, it may not be the optimal mechanism for extensive search of a large surface comparable to that of the entire cell membrane. Loverdo et al. [56] suggested that reversibly jumping between 3D and 1D may optimize transport over long distances. These authors were considering active transport in 1D, although not necessarily directional, and very slowly diffusing objects, such as intracellular vesicles. The underlying principle is that combining slow and fast random motions can optimize the search of large regions of space.

\section{Conclusions}

In this perspective article, I have has tried to present, from a biophysical point of view, possibly relevant elements helping to understand the important role of signaling peripheral 
membrane proteins, such as Src or Ras, which share a labile lipid anchor (myristoyl or farnesyl) and clusters of alternating basic and neutral residues.

I speculate that these biological features exploit the dimensionality reduction in the cell boundary by making use of the capacity to switch between a mobile population of monomers that can not only detach from the plasma membrane to the cytoplasm but can also rapidly diffuse in the juxtamembrane space, and an immobile fraction of active dimers, in the case of Src.

I have also highlighted the multiple roles of an excess of basic over acidic residues in intrinsically disordered regions tethered to membranes either by lipid anchors or by transmembrane helices (such as in RTK): (i) enhancing binding to negatively charged membranes, (ii) recruiting low abundance acidic lipids, (iii) mediating protein self- and hetero association through acidic lipid-mediated charge-bridges, or (iv) promoting direct protein-protein contacts by concentrating and orienting segments with alternating charges to form membrane-tethered $b$-sheets.

We are well aware that the overwhelming complexity of cell signaling goes far beyond this reductionist view. On the other hand, the complexity should not drive us away from seeking to identify the intrinsic simplicity underlaying the physical chemistry of life.

Funding: This research was funded by the Spanish Ministry of Science and Innovation (PID2019104914RB-I00) and Fundación Maria Francisca de Roviralta.

Acknowledgments: I want to acknowledge the extensive discussions and insight provided by Irrem-Laareb Mohammad and Anabel-Lise Le Roux as well as other members of the Biomolecular NMR group.

Conflicts of Interest: The author declares no conflict of interest.

\section{References}

1. Symons, J.; Cho, K.-J.; Chang, J.T.; Du, G.; Waxham, M.N.; Hancock, J.F.; Levental, I.; Levental, K.R. Lipidomic atlas of mammalian cell membranes reveals hierarchical variation induced by culture conditions, subcellular membranes, and cell lineages. Soft Matter 2021, 17, 288-297. [CrossRef]

2. Mohammad, I.-L.; Mateos, B.; Pons, M. The disordered boundary of the cell: Emerging properties of membrane-bound intrinsically disordered proteins. Biomol. Concepts 2019, 10, 25-36. [CrossRef] [PubMed]

3. Wang, M.; Weiss, M.; Simonovic, M.; Haertinger, G.; Schrimpf, S.P.; Hengartner, M.O.; von Mering, C. PaxDb, a database of protein abundance averages across all three domains of life. Mol. Cell. Proteom. 2012, 11, 492-500. [CrossRef] [PubMed]

4. Cornish, J.; Chamberlain, S.G.; Owen, D.; Mott, H.R. Intrinsically disordered proteins and membranes: A marriage of convenience for cell signalling? Biochem. Soc. Trans. 2020, 48, 2669-2689. [CrossRef] [PubMed]

5. Burn, P. Talking point Amphitropic proteins: A new class of membrane proteins. Trends Biochem. Sci. 1988, 13, 79-83. [CrossRef]

6. Nastou, K.; Tsaousis, G.N.; Kremizas, K.E.; Litou, Z.I.; Hamodrakas, S.J. The human plasma membrane peripherome: Visualization and analysis of interactions. BioMed Res. Int. 2014, 2014, 1-12. [CrossRef]

7. Lomize, M.A.; Lomize, A.L.; Pogozheva, I.D.; Mosberg, H.I. OPM: Orientations of proteins in membranes database. Bioinformatics 2006, 22, 623-625. [CrossRef] [PubMed]

8. Allen, K.N.; Entova, S.; Ray, L.C.; Imperiali, B. Monotopic Membrane Proteins Join the Fold. Trends Biochem. Sci. 2019, 44, 7-20. [CrossRef]

9. Resh, M.D. Trafficking and signaling by fatty-acylated and prenylated proteins. Nat. Chem. Biol. 2006, 2, 584-590. [CrossRef]

10. Mosior, M.; McLaughlin, S. Binding of Basic Peptides to Acidic Lipids in Membranes: Effects of Inserting Alanine(s) Between the Basic Residues. Biochemistry 1992, 31, 1767-1773. [CrossRef]

11. McLaughlin, S. The electrostatic properties of membranes. Annu. Rev. Biophys. Biophys. Chem. 1989, 18, 113-136. [CrossRef]

12. Balach, M.M.; Casale, C.H.; Campetelli, A.N. Erythrocyte plasma membrane potential: Past and current methods for its measurement. Biophys. Rev. 2019, 11, 995-1005. [CrossRef]

13. Gurtovenko, A.A.; Vattulainen, I. Lipid transmembrane asymmetry and intrinsic membrane potential: Two sides of the same coin. J. Am. Chem. Soc. 2007, 129, 5358-5359. [CrossRef] [PubMed]

14. Gairí, M.; Romi, R.; Fernández, I.; Rochat, H.; Martin-Eauclaire, M.F.; Van Rietschoten, J.; Pons, M.; Giralt, E. 3D structure of kaliotoxin: Is residue 34 a key for channel selectivity? J. Pept. Sci. 1997, 3, 314-319. [CrossRef]

15. Weber, D.; Yao, S.; Rojko, N.; Anderluh, G.; Lybrand, T.P.; Downton, M.T.; Wagner, J.; Separovic, F. Characterization of the lipid-binding site of equinatoxin II by NMR and molecular dynamics simulation. Biophys. J. 2015, 108, 1987-1996. [CrossRef] [PubMed] 
16. Khan, H.M.; He, T.; Fuglebakk, E.; Grauffel, C.; Yang, B.; Roberts, M.F.; Gershenson, A.; Reuter, N. A Role for Weak Electrostatic Interactions in Peripheral Membrane Protein Binding. Biophys. J. 2016, 110, 1367-1378. [CrossRef]

17. Branduardi, D.; Gervasio, F.L.; Cavalli, A.; Recanatini, A.M.; Parrinello, M. The role of the peripheral anionic site and cation $-\pi$ interactions in the ligand penetration of the human AChE gorge. J. Am. Chem. Soc. 2005, 127, 9147-9155. [CrossRef]

18. Zhenyu, L.; Lai, J.; Yingkai, Z. Importance of charge independent effects in readout of the trimethyllysine mark by HP1 chromodomain. J. Am. Chem. Soc. 2009, 131, 14928-14931.

19. Gianni, S.; Freiberger, M.I.; Jemth, P.; Ferreiro, D.U.; Wolynes, P.G.; Fuxreiter, M. Fuzziness and Frustration in the Energy Landscape of Protein Folding, Function, and Assembly. Acc. Chem. Res. 2021, 54, 1251-1259. [CrossRef]

20. McLaughlin, S.; Aderem, A. The myristoyl-electrostatic switch: A modulator of reversible protein-membrane interactions. Trends Biochem. Sci. 1995, 20, 272-276. [CrossRef]

21. Kim, J.; Mosior, M.; Chung, L.; Wu, H.; McLaughlin, S. Binding of peptides with basic residues to membranes containing acidic phospholipids. Biophys. J. 1991, 60, 135-148. [CrossRef]

22. Ben-Tal, N.; Honig, B.; Peitzsch, R.; Denisov, G.; McLaughlin, S. Binding of small basic peptides to membranes containing acidic lipids: Theoretical models and experimental results. Biophys. J. 1996, 71, 561-575. [CrossRef]

23. Deryusheva, E.; Nemashkalova, E.; Galloux, M.; Richard, C.-A.; Eléouët, J.-F.; Kovacs, D.; Van Belle, K.; Tompa, P.; Uversky, V.N.; Permyakov, S. Does Intrinsic Disorder in Proteins Favor Their Interaction with Lipids? Proteom. 2019, 19, e1800098. [CrossRef]

24. Piovesan, D.; Tabaro, F.; Mičetić, I.; Necci, M.; Quaglia, F.; Oldfield, C.J.; Aspromonte, M.C.; Davey, N.E.; Davidovic, R.; Dosztanyi, Z.; et al. DisProt 7.0: A major update of the database of disordered proteins. Nucleic Acids Res. 2017, 45, D219-D227. [CrossRef]

25. Resh, M.D. Fatty acylation of proteins: New insights into membrane targeting of myristoylated and palmitoylated proteins. Biochim. Biophys. Acta Mol. Cell Res. 1999, 1451, 1-16. [CrossRef]

26. Castrec, B.; Dian, C.; Ciccone, S.; Ebert, C.L.; Bienvenut, W.V.; Le Caer, J.-P.; Steyaert, J.-M.; Giglione, C.; Meinnel, T. Structural and genomic decoding of human and plant myristoylomes reveals a definitive recognition pattern. Nat. Chem. Biol. 2018, 14, 671-679. [CrossRef]

27. Charron, G.; Li, M.M.H.; MacDonald, M.R.; Hang, H.C. Prenylome profiling reveals S-farnesylation is crucial for membrane targeting and antiviral activity of ZAP long-isoform. Proc. Natl. Acad. Sci. USA 2013, 110, 11085-11090. [CrossRef]

28. McLaughlin, S.; Murray, D. Plasma membrane phosphoinositide organization by protein electrostatics. Nature 2005, $438,605-611$. [CrossRef]

29. Michailidis, I.E.; Rusinova, R.; Georgakopoulos, A.; Chen, Y.; Iyengar, R.; Robakis, N.K.; Logothetis, D.E.; Baki, L. Phosphatidylinositol-4,5-bisphosphate regulates epidermal growth factor receptor activation. Pflügers Arch. Eur. J. Physiol. 2011, 461, 387-397. [CrossRef] [PubMed]

30. Arkhipov, A.; Shan, Y.; Das, R.; Endres, N.F.; Eastwood, M.P.; Wemmer, D.E.; Kuriyan, J.; Shaw, D.E. Architecture and membrane interactions of the EGF receptor. Cell 2013, 152, 557-569. [CrossRef]

31. Halim, K.B.A.; Koldsø, H.; Sansom, M.S. Interactions of the EGFR juxtamembrane domain with PIP2-containing lipid bilayers: Insights from multiscale molecular dynamics simulations. Biochim. Biophys. Acta Gen. Subj. 2015, 1850, 1017-1025. [CrossRef] [PubMed]

32. Hedger, G.; Sansom, M.S.P.; Koldsø, H. The juxtamembrane regions of human receptor tyrosine kinases exhibit conserved interaction sites with anionic lipids. Sci. Rep. 2015, 5, 9198. [CrossRef] [PubMed]

33. Van Den Bogaart, G.; Meyenberg, K.; Risselada, H.J.; Amin, H.; Willig, K.I.; Hubrich, B.E.; Dier, M.; Hell, S.W.; Grubmuller, H.; Diederichsen, U.; et al. Membrane protein sequestering by ionic protein-lipid interactions. Nature 2011, 479, 552-555. [CrossRef]

34. Hädicke, A.; Blume, A. Binding of cationic model peptides (KX) $4 \mathrm{~K}$ to anionic lipid bilayers: Lipid headgroup size influences secondary structure of bound peptides. Biochim. Biophys. Acta Biomembr. 2017, 1859, 415-424. [CrossRef]

35. Sinthuvanich, C.; Veiga, A.S.; Gupta, K.; Gaspar, D.; Blumenthal, R.; Schneider, J.P. Anticancer $\beta$-hairpin peptides: Membraneinduced folding triggers activityc. J. Am. Chem. Soc. 2012, 134, 6210-6217. [CrossRef]

36. Almo, S.C.; Bonanno, J.B.; Sauder, J.M.; Emtage, S.; DiLorenzo, T.P.; Malashkevich, V.; Wasserman, S.R.; Swaminathan, S.; Eswaramoorthy, S.; Agarwal, R.; et al. Structural genomics of protein phosphatases. J. Struct. Funct. Genom. 2007, 8, 121-140. [CrossRef] [PubMed]

37. Le Roux, A.L.; Busquets, M.A.; Sagués, F.; Pons, M. Kinetics characterization of c-Src binding to lipid membranes: Switching from labile to persistent binding. Colloids Surf. B Biointerfaces 2016, 138, 17-25. [CrossRef]

38. Le Roux, A.L.; Castro, B.; Garbacik, E.T.; Garcia Parajo, M.F.; Pons, M. Single molecule fluorescence reveals dimerization of myristoylated Src N-terminal region on supported lipid bilayers. ChemistrySelect 2016, 1, 642-647. [CrossRef]

39. Li, S.; Couet, J.; Lisanti, M.P. Src tyrosine kinases, G( $\alpha$ ) subunits, and H-Ras share a common membrane-anchored scaffolding protein, caveolin: Caveolin binding negatively regulates the auto-activation of Src tyrosine kinases. J. Biol. Chem. 1996, 271, 29182-29190. [CrossRef]

40. Barker, S.; Kassel, D.B.; Weigl, D.; Huang, X.; Luther, M.; Knight, W.B. Characterization of pp60c-src Tyrosine Kinase Activities Using a Continuous Assay: Autoactivation of the Enzyme Is an Intermolecular Autophosphorylation Process. Biochemistry 1995, 34, 14843-14851. [CrossRef]

41. Le Roux, A.L.; Mohammad, I.L.; Mateos, B.; Arbesú, M.; Gairí, M.; Khan, F.A.; Farman, A.; Teixeira, J.; Pons, M. A MyristoylBinding Site in the SH3 Domain Modulates c-Src Membrane Anchoring. iScience 2019, 12, 194-203. [CrossRef] [PubMed] 
42. Adam, G.; Delbrück, M. Reduction of Dimensionality in Biological Diffusion Processes. In Structural Chemistry and Molecular Biology; Rich, A., Davidson, N., Eds.; W.H. Freeman and Co.: San Francisco, CA, USA, 1968.

43. Berg, O.G.; Winter, R.B.; Von Hippel, P.H. Diffusion-Driven Mechanisms of Protein Translocation on Nucleic Acids. 1. Models and Theory. Biochemistry 1981, 20, 6929-6948. [CrossRef] [PubMed]

44. Cherry, R.J. Rotational and lateral diffusion of membrane proteins. Biochim. Biophys. Acta Rev. Biomembr. 1979, 559, $289-327$. [CrossRef]

45. Kholodenko, B.N.; Brown, G.C.; Hoek, J.B. Diffusion control of protein phosphorylation in signal transduction pathways. Biochem. J. 2000, 350, 901-907. [CrossRef]

46. Kholodenko, B.N.; Hoek, J.; Westerhoff, H. Why cytoplasmic signalling proteins should be recruited to cell membranes. Trends Cell Biol. 2000, 10, 173-178. [CrossRef]

47. Saffman, P.G.; Delbruck, M. Brownian motion in biological membranes. Proc. Natl. Acad. Sci. USA 1975, 72, 3111-3113. [CrossRef]

48. Hughes, B.D.; Pailthorpe, B.A.; White, L.R. The Translational and Rotational Drag on a Cylinder Moving in a Membrane. J. Fluid Mech. 1981, 110, 349-372. [CrossRef]

49. Petrov, E.P.; Schwille, P. Translational diffusion in lipid membranes beyond the Saffman-Delbrück approximation. Biophys. J. 2008, 94, L41-L43. [CrossRef]

50. Goutaland, Q.; Fournier, J.-B. Saffman-Delbrück and beyond: A pointlike approach. Eur. Phys. J. E 2019, 42, 156. [CrossRef]

51. Javanainen, M.; Martinez-Seara, H.; Metzler, R.; Vattulainen, I. Diffusion of Integral Membrane Proteins in Protein-Rich Membranes. J. Phys. Chem. Lett. 2017, 8, 4308-4313. [CrossRef]

52. Ziemba, B.P.; Falke, J.J. Lateral diffusion of peripheral membrane proteins on supported lipid bilayers is controlled by the additive frictional drags of (1) bound lipids and (2) protein domains penetrating into the bilayer hydrocarbon core. Chem. Phys. Lipids 2013, 172-173, 67-77. [CrossRef]

53. Nawrocki, G.; Im, W.; Sugita, Y.; Feig, M. Clustering and dynamics of crowded proteins near membranes and their influence on membrane bending. Proc. Natl. Acad. Sci. USA 2019, 116, 24562-24567. [CrossRef]

54. Schleicher, K.D.; Dettmer, S.L.; Kapinos, L.E.; Pagliara, S.; Keyser, U.; Jeney, S.; Lim, R. Selective transport control on molecular velcro made from intrinsically disordered proteins. Nat. Nanotechnol. 2014, 9, 525-530. [CrossRef]

55. Sil, P.; Mateos, N.; Nath, S.; Buschow, S.; Manzo, C.; Suzuki, K.G.N.; Fujiwara, T.; Kusumi, A.; Garcia-Parajo, M.F.; Mayor, S. Dynamic actin-mediated nano-scale clustering of CD44 regulates its meso-scale organization at the plasma membrane. Mol. Biol. Cell 2020, 31, 561-579. [CrossRef]

56. Loverdo, C.; Bénichou, O.; Moreau, M.; Voituriez, R. Enhanced reaction kinetics in biological cells. Nat. Phys. 2008, 4, 134-137. [CrossRef] 